\title{
Why involve consumers in eye health research?
}

K Twamley , J Evans ${ }^{2}$ and R Wormald ${ }^{2,3}$

Eye (2011) 25, 969-970; doi:10.1038/eye.2011.133

Although the aim of research into the prevention and treatment of eye disease is to improve the health and quality of life of patients, we seldom involve people affected by eye disease ('consumers') in the decisions that we make when designing and conducting research. A recent review of the literature by the James Lind Alliance (http://www.lindalliance.org/), found only one published example in eye health. ${ }^{1}$ In contrast, consumer participation is well established in other areas of health research, most notably in HIV/AIDS. Patient and public involvement has been endorsed by many governments, including the USA, ${ }^{2}$ the UK, ${ }^{3}$ Australia, ${ }^{4}$ and by the World Health Organization. ${ }^{5}$ It is increasingly required by funding and research agencies. ${ }^{6}$

Clinical research studies are often designed by clinicians who feel that they understand their patients' needs and concerns. But there is evidence that consumers have different research priorities. ${ }^{7,8}$ Failure to engage with them can result in a research agenda biased towards academic and industry interests. For example, a study on treatments for osteoarthritis of the knee found that consumers had a preference for conservative treatments, such as physiotherapy, and wanted more research on self help and education; the overwhelming majority of published studies were about drug and surgical treatments. $^{8}$

Our experience with a consumer panel for age-related macular degeneration (AMD) highlights some of the benefits of collaboration. The model we used is based on experience from The Cochrane Collaboration. Volunteers were recruited through an advertisement in an AMD patient support group magazine. All the members of the panel either have AMD (with differing levels of visual impairment), or care for a family relative with the condition. Every month a review summary is sent by email or letter to these volunteers for comment. To date, the panel has looked at 14 review summaries of interventions for AMD and low vision. The format is open-ended and allows for any comment to be made. As expected, the panel picked up many instances where lack of clarity or unnecessary use of jargon made the research summary difficult for consumers to understand.

The panels have also expressed their opinions on the nature of the research undertaken.

A particular issue arose with the review of 'Reading aids for low vision'. 9 The plain language summary of this review states 'Results from small studies of unclear quality and weak design (...) were inconclusive'. The members of the panel were both shocked and disappointed that such an important question for them had apparently been so poorly addressed by the scientific community. Consumers' accounts of difficulties in finding the 'right' vision aid made a strong case for more research in this area. One participant commented: 'It is important to me, because I could not operate without sophisticated electronic aids, two of which I use all the time. Simple optical magnifiers of different types are of no help to me in reading. Our own Low Vision Clinic is excellent, but seems to deal only with conventional optical aids'. It is clearly a complex area of study, with many different types of interventions available and new ones emerging all the time. There are numerous different potential outcomes, depending on the needs of the individual. Nonetheless, these problems are not insurmountable and, without engaging with consumers, may never be properly dealt with. Given the impact on the lives of people with low vision, the topic merits more time and attention.

We also asked the panel for input into the choice of outcomes for an overview of systematic reviews of interventions for neovascular AMD. ${ }^{10}$ Their involvement resulted in important changes to the outcomes considered in the review. Two outcomes were
${ }^{1}$ Social Science Research Unit, Institute of Education, London, UK

${ }^{2}$ International Centre for Eye Health, London School of Hygiene and Tropical Medicine, London, UK

${ }^{3}$ Moorfields Eye Hospital, London, UK

Correspondence: J Evans, International Centre for Eye Health, London School of Hygiene and Tropical Medicine, Keppel Street, London WC1E 7HT, UK Tel: + 44 (0)20 7958 8167; Fax: + 44 (0)20 74364230 E-mail: jennifer.evans@ Ishtm.ac.uk 
identified that are important to consumers, but that are rarely reported in the publication of existing randomised controlled trials. Consumers want to know when making decisions about treatment options what is the risk of 'going completely blind,' ${ }^{1}$ and what is the chance of achieving vision good enough for driving a car and reading? ${ }^{10}$

We have to provide relevant information to our patients on the benefits and harms of treatment so that they can make properly informed (evidence-based) choices. Consumer involvement in the design of research ensures that the questions being asked and the outcomes investigated are those that matter to the very people who suffer from vision loss and eye disease. Ultimately, this will be of value to practicing ophthalmologists who will have evidence on which to base the answers to their patients' questions.

A key feature of consumer involvement is that it entails research with, not on, patients or consumers. ${ }^{11}$ This means co-opting consumers as colleagues in research groups; they are colleagues that have a particular expertise, that of living with the condition and possibly undergoing treatment for it. We have found that such collaboration has helped to sustain enthusiasm and on-going involvement; the AMD consumer panel has been active for over 18 months. It has also been mutually educational for researchers and consumers as has been found with other groups. ${ }^{12}$ A concern of some researchers is that consumers are self-selected and may have a specific agenda or complaint that they wish to promulgate. This can happen, of course, but also applies to research scientists; hence, the need for conflict of interest statements. The involvement of several different members of a consumer advisory panel should help control excessively dominant opinions.

The greatest challenge with involving consumers in research is to actually act on the result of discussions. In setting out our experiences with the AMD consumer panel, this article is part of that process. These two brief examples show how our collaboration with consumers in the research process has given us valuable information. We are now more confident that we are researching what matters.

The patient doctor relationship has evolved. No longer are patients and public willing to accept without question their doctors' treatment suggestion. ${ }^{13}$ Faced with sometimes conflicting advice from doctors, consumers look to the internet to find answers for their treatment questions. Although some may access databases such as PubMed and peer-reviewed scientific journals, others will access patient forums in search of information that suits their needs. It is time that we take the initiative to ensure that everyone has access to information that is accurate and relevant.

\section{Conflict of interest}

The authors declare no conflict of interest.

\section{Acknowledgements}

All members of the Cochrane Eyes and Vision Group consumer panels, in particular David Brandon, Harold Burton, Angie Butler, Bill Crowther, Peter Dyson, Jan Elson, Diana Fitchett and Brenda Rogers who read and made comments on a draft of this editorial.

\section{References}

1 Margo CE, Mack WP. Therapeutic decisions involving disparate clinical outcomes: patient preference survey for treatment of central retinal artery occlusion. Ophthalmology 1996; 103(4): 691-696.

2 Public Law 89-749. Comprehensive Health Planning and Public Health Service Amendments of 1966. 3 November 1966 (P.L. 88-164, P.L. 89-749, and P.L. 93-641).

3 Department of Health. Patient and Public Involvement in the NHS. Department of Health: London, 1999.

4 NHMRC and CHF. Statement on Consumer and Community Participation in Health and Medical Research, Commonwealth of Australia. National Health and Medical Research Council, Government of Australia, 2002.

5 WHO. Declaration of Alma-Ata. In: International Conference on Primary Health Care, Alma-Ata, USSR 1978.

6 NIHR. Research for Patient benefit (RfPB) Programme Guidelines for Applicants. National Institute for Health Research: London, 2009.

7 Stewart R, Caird J, Oliver K, Oliver S. Clinicians' and Patients' Research Priorities: A Scoping Review. 17th Cochrane Colloquim, Singapore, 2009.

8 Tallon D, Chard J, Dieppe P. Relation between agendas of the research community and the research consumer. Lancet 2000; 355(9220): 2037-2040.

9 Virgili G, Acosta R. Reading aids for adults with low vision. Cochrane Database Syst Rev 2006; (4): CD003303.

10 Evans JR, Virgili G, Gordon I, Bunce C, Chakravarthy U, Desai $\mathrm{P}$ et al. Interventions for neovascular age-related macular degeneration (Protocol). The Cochrane Library 2009; (4).

11 Boote J, Telford R, Cooper C. Consumer involvement in health research: a review and research agenda. Health Policy 2002; 61(2): 213-236.

12 Oliver SR, Rees RW, Clarke-Jones L, Milne R, Oakley AR, Gabbay J et al. A multidimensional conceptual framework for analysing public involvement in health services research. Health Expect 2008; 11(1): 72-84.

13 Hibbard J, Weeks E. Consumerism in health care; prevalence and predictors. Medical Care 1987; 25: 1019-1033. 